\title{
Generalized entropies under different probability normalization conditions
}

\author{
OU CongJie ${ }^{1 *} \&$ CHEN JinCan ${ }^{2}$ \\ ${ }^{1}$ College of Information Science and Engineering, Huaqiao University, Xiamen 361021, China; \\ ${ }^{2}$ Department of Physics, Xiamen University, Xiamen 361005, China
}

Received May 17, 2011; accepted July 20, 2011

\begin{abstract}
Tsallis entropy and incomplete entropy are proven to have equivalent mathematical structure except for one nonextensive factor $q$ through variable replacements on the basis of their forms. However, employing the Lagrange multiplier method, it is judged that neither yields the $q$-exponential distributions that have been observed for many physical systems. Consequently, two generalized entropies under complete and incomplete probability normalization conditions are proposed to meet the experimental observations. These two entropic forms are Lesche stable, which means that both vary continuously with probability distribution functions and are thus physically meaningful.
\end{abstract}

generalized entropy, $q$-exponential distribution, incomplete probability normalization, Lesche stability

Citation: Ou C J, Chen J C. Generalized entropies under different probability normalization conditions. Chinese Sci Bull, 2011, 56: 3649-3653, doi: 10.1007/ s11434-011-4809-0

The concept of entropy can be traced from the second law of thermodynamics, which determines the available energy in a thermodynamic system. Entropy has been widely used in different fields, such as the solar thermophotovoltaic system [1], nonequilibrium system [2], and in the design of multistage heat engine systems [3]. In recent years, the generalization of classical Boltzmann-Gibbs entropy has become a powerful tool for handling physical systems with long-range interaction [4,5], long-duration memory [6], nonequilibrium dynamics [7], and fractal phase-space structures [8]. Ideal assumptions, such as those of free particles and infinite systems, are not suitable for more and more systems with the development of modern physics. Among the different generalizations of statistical physics theory, the nonextensive method proposed by Tsallis [9] in 1988 has become the most well known. The kernel of the Tsallis nonextensive statistical method is the q-generalized entropy, which can be written as

*Corresponding author (email: jcou@ @qu.edu.cn)

$$
S_{q}=k \frac{1-\sum_{i=1}^{W} p_{i}^{q}}{q-1},
$$

where $q$ is the nonextensive index, $k$ is the Boltzmann constant and $p_{i}$ is the probability of the $i$ th microstate among all $W$ microstates of the system. Obviously, eq. (1) reduces to classical Boltzmann-Gibbs entropy at the $q \rightarrow 1$ limit. It is worth mentioning that in the framework of the Tsallis statistics, probability normalization is the same as that in the traditional framework. Hence, the distribution of the random variable of the system is based on complete information. For instance, if we consider the position of a particle confined in finite space, all possible values of the position are known and the information of the position is complete. However, for some physical systems presenting many complex interactions, some values of position are not known exactly or cannot be written analytically, and thus, we cannot obtain all possible states of the systems. In this case, information of the system is incomplete. On this basis, 
Wang [10] proposed another nonextensive statistical method referred to as "incomplete statistics" in 2001. The entropic form of incomplete statistics can be written as

$$
S_{I}=k \frac{\sum_{i=1}^{W^{\prime}} p_{i}^{\prime}-1}{q^{\prime}-1},
$$

where $p_{i}^{\prime}$ is the probability of the $i$ th microstate among the $W^{\prime}$ accessible states and here $W^{\prime}$ is no longer the total number of possible states $\left(W_{T}\right)$ of the system because of the incomplete probability normalization, $\sum_{i=1}^{W^{\prime}}\left(p_{i}^{\prime}\right)^{q^{\prime}}=1$. Thus, the nonextensive index $q^{\prime}$ should be a nonnegative real number. This kind of nonextensive generalization of classical theory is suitable for the analysis of multi-fractal phase space. It has been proved that $q^{\prime}$ is the ratio between the fractal Hausdorff dimension and Euclidean dimension [8]. Tsallis statistics and incomplete statistics are two important nonextensive methods for dealing with nonideal systems that have complete information and incomplete information, respectively. However, the mathematical structures of these two entropies are very similar.

\section{Mathematical comparability of Tsallis entropy and incomplete entropy}

If we compare the probability normalization of the entropic forms of eqs. (1) and (2), it is easy to find a variable relation between $p_{i}$ and $p_{i}{ }^{\prime}$ :

$$
\sum_{i=1}^{W} p_{i}=\sum_{i=1}^{W^{\prime}}\left(p_{i}^{\prime}\right)^{q^{\prime}}=1
$$

From eq. (3), if the variable replacement

$$
\left\{\begin{array} { l } 
{ p _ { i } = ( p _ { i } ^ { \prime } ) ^ { q ^ { \prime } } } \\
{ q = 1 / q ^ { \prime } }
\end{array} \text { or } \quad \left\{\begin{array}{l}
p_{i}^{\prime}=p_{i}^{q} \\
q^{\prime}=1 / q
\end{array}\right.\right.
$$

is adopted, we can obtain the mathematical relations between the Tsallis entropy and incomplete entropy in symmetrical form:

$$
\left\{\begin{array}{l}
S_{q}=q^{\prime} S_{I}, \\
S_{I}=q S_{q} .
\end{array}\right.
$$

It is directly seen from eq. (5) that the Tsallis entropy and incomplete entropy are mathematically equivalent except for a factor $q$ (or $\left.q^{\prime}\right)$. From a physical point of view, the information on microstates of a system can be either complete or incomplete, but never both. This means that the Tsallis statistics and incomplete statistics cannot be used for the same system. Nevertheless, it has been proved that incomplete statistical mechanics has theoretical characteristics similar to those of Tsallis statistical mechanics [10]. Max- imizing the incomplete entropy within the constraints of incomplete probability normalization and incomplete energy expectation employing the Lagrange multiplier method yields [11]

$$
\left\{\begin{array}{l}
p_{i}^{\prime}=\frac{1}{Z}\left[1-\beta\left(1-q^{\prime}\right) q^{\prime} \frac{\varepsilon_{i}-U}{\sum_{j} p_{j}^{\prime}}\right]^{\frac{1}{1-q^{\prime}}}, \\
Z=\left\{\sum_{i}\left[1-\beta\left(1-q^{\prime}\right) q^{\prime} \frac{\varepsilon_{i}-U}{\sum_{j} p_{j}^{\prime}}\right]^{\frac{q^{\prime}}{1-q^{\prime}}}\right\}^{1 / q^{\prime}},
\end{array}\right.
$$

where $\beta$ is the Lagrange multiplier associated with the energy constraint, $\varepsilon_{i}$ is the energy of the $i$ th microstate, and $U^{\prime}=\sum_{i}\left(p_{i}^{\prime}\right)^{q^{\prime}} \varepsilon_{i}$ is the internal energy.

The Lagrange multiplier method can also be applied to Tsallis entropy to yield the probability distribution function. In this case, the probability normalization is based on a complete information ensemble; i.e. $\sum_{i} p_{i}=1$. Meanwhile, there are three choices for the energy expectation constraint [12]. Comparing the frameworks of Tsallis statistics and incomplete statistics with consideration of eq. (4), we find that the first energy constraint $\left(U=\sum_{i} p_{i} \varepsilon_{i}\right)$ is the most reasonable. Thus, maximizing eq. (1) within the constraints of probability normalization and the first choice of energy expectation yields [13]

$$
\left\{\begin{array}{l}
p_{i}=\frac{1}{Z}\left[1-\beta \frac{q-1}{q} \frac{\varepsilon_{i}-U}{\sum_{j} p_{j}^{q}}\right]^{\frac{1}{q-1}}, \\
Z=\sum_{i}\left[1-\beta \frac{q-1}{q} \frac{\varepsilon_{i}-U}{\sum_{j} p_{j}^{q}}\right]^{\frac{1}{q-1}} .
\end{array}\right.
$$

Here we do not go further to consider the other two energy constraints. The reasons are as follows. The second choice of the energy constraint $\left(U=\sum_{i} p_{i}^{q} \varepsilon_{i}\right)$ conflicts with the probability normalization because of an obvious mathematical inequality, $\quad \sum_{i} p_{i}^{q} \neq \sum_{i} p_{i}=1, \quad(q \neq 1)$. The third choice of the energy constraint $U=\sum_{i} p_{i}^{q} \varepsilon_{i} / \sum_{j} p_{j}^{q}$ is not Lesche stable [14] for small deformations of the probability distribution function.

Employing the standard Lagrange multiplier method, the results of maximizing incomplete entropy and Tsallis entropy may have other forms. They can be respectively written as 


$$
p_{i}^{\prime}=\left[1-\left(1-q^{\prime}\right) q^{\prime}\left(x_{i}+\alpha+\frac{1}{\left(1-q^{\prime}\right) q^{\prime}}\right)\right]^{\frac{1}{1-q^{\prime}}}
$$

and

$$
p_{i}=\left[1-\frac{q-1}{q}\left(x_{i}+\alpha+\frac{q}{q-1}\right)\right]^{\frac{1}{q-1}},
$$

where $x_{i}=\beta \varepsilon_{i}$ and $\alpha$ is the Lagrange multiplier associated with the constraint of probability normalization. Comparing eqs. (6) and (8) (or eqs. (7) and (9)), one finds that it is possible to set $Z=1$ by applying a uniform spectrum shift $x_{i} \rightarrow x_{i}+\alpha+1 /\left[\left(1-q^{\prime}\right) q^{\prime}\right]$ (or $\left.x_{i} \rightarrow x_{i}+\alpha+q /(q-1)\right)$.

By contrast, it has been observed in more and more physical systems having long-range interaction and/or long-duration memory that the probability distribution function deviates from the classical distribution function and can be written as

$$
p_{i} \propto\left[1-(q-1) x_{i}\right]^{\frac{1}{q-1}}
$$

The long-range interaction systems mentioned before including two-dimensional turbulence [15], pure electron plasma $[16,17]$, self-gravitating galaxy and so on. It is possible to rewrite eq. (10) as

$$
p_{i}=\left[1-(q-1)\left(x_{i}+a\right)\right]^{\frac{1}{q-1}}
$$

using the same technique of the spectrum shift [18], where $a$ is a real number. Eq. (11) is obviously not equivalent to eqs. (8) and (9) implying that the results of maximizing Tsallis entropy (or incomplete entropy) have nothing to do with the observed phenomena of real physical systems. To investigate the thermo-statistical characteristics of such abnormal systems, a new kind of generalized entropy is needed.

\section{Properties of generalized entropies}

Employing the VarEntropy method [19], we recently proposed a generalized entropic form [18] that can be traced from the first and second laws of thermodynamics. The generalized entropic form can be written as

$$
\tilde{S}_{q}=\frac{1-\sum_{i=1}^{W} p_{i}^{q}}{q(q-1)} \quad(q>0),
$$

where we set the Boltzmann constant $k=1$ for the sake of convenience. The steps for deriving this new kind of entropy are exactly the reverse of those of the MaxEnt method. Thus, it is natural that this generalized entropy yields exactly the observed $q$-exponential function. Other character- istics, such as nonadditivity and concavity, are discussed in [18]. It is shown that this generalized entropy is more suitable for describing systems having long-range interactions and/or long-duration memory.

As mentioned above, it is possible for a physical system to have incomplete information because of the complexity of the internal interactions. In this case, the probability normalization is incomplete; i.e. $\sum_{i=1}^{W^{\prime}}\left(p_{i}^{\prime}\right)^{q^{\prime}}=1$. For this kind of probability normalization, we can also derive the generalized entropic form employing the VarEntropy method. We start from the observed $q$-exponential function

$$
p_{i}^{\prime}=\left[1-\left(1-q^{\prime}\right)\left(x_{i}+a\right)\right]^{\frac{1}{1-q^{\prime}}},
$$

which is the same as eq. (11) if $q=2-q^{\prime}$ is adopted. From eq. (13), we obtain

$$
x_{i}=\frac{1-\left(p_{i}^{\prime}\right)^{1-q^{\prime}}}{1-q^{\prime}}-a .
$$

The variation in entropy can be written as

$$
\mathrm{d} \tilde{S}_{I}=\mathrm{d} \bar{x}-\overline{\mathrm{d} x}=\sum_{i=1}^{W^{\prime}} x_{i} \mathrm{~d}\left(p_{i}^{\prime}\right)^{q^{\prime}}
$$

Substituting eq. (14) into eq. (15) yields

$$
\mathrm{d} \tilde{S}_{I}=\sum_{i=1}^{W^{\prime}}\left(\frac{-\left(p_{i}^{\prime}\right)^{1-q^{\prime}}}{1-q^{\prime}}\right) \mathrm{d}\left(p_{i}^{\prime}\right)^{q^{\prime}} .
$$

We then obtain the generalized entropy under incomplete probability normalization as

$$
\begin{aligned}
\tilde{S}_{I} & =\sum_{i=1}^{W^{\prime}} \int\left(\frac{-\left(p_{i}^{\prime}\right)^{1-q^{\prime}}}{1-q^{\prime}}\right) q^{\prime}\left(p_{i}^{\prime}\right)^{q^{\prime}-1} \mathrm{~d} p_{i}^{\prime} \\
& =\frac{q^{\prime}\left(\sum_{i} p_{i}^{\prime}-C\right)}{q^{\prime}-1},
\end{aligned}
$$

where $C$ is the integral constant. In the case of a system for which the probability distribution function satisfies $p_{i}^{\prime}=\delta_{1, i}$, the entropy should be zero, and thus $C=1$. Hence, the incomplete generalized entropy can be written as

$$
\tilde{S}_{I}=\frac{q^{\prime}\left(\sum_{i=1}^{W^{\prime}} p_{i}^{\prime}-1\right)}{q^{\prime}-1} \quad\left(q^{\prime}>0\right) .
$$

Eqs. (12) and (18) show that generalized entropies under complete and incomplete probability normalizations differ from Tsallis entropy and incomplete entropy, respectively.

On the basis of eqs. (12) and (18), we can discuss the Lesche stability of the generalized entropies for different probability normalizations. For a given statistical mechanical system, the probability distribution can be obtained through experimental measures. Consequently, the entropy 
can be determined since it is a function of the probability distribution. Two measurements campaign to establish the probability distribution for a given system should produce similar results. It thus seems reasonable that the entropy values of the two probability distributions would also be similar. For complete probability normalization, the definition of Lesche stability reads

$$
\begin{aligned}
& (\forall \varepsilon>0)(\exists \delta>0) \\
& \left(\begin{array}{l}
\left\|p^{\langle 1\rangle}-p^{\langle 2\rangle}\right\|_{1} \leqslant \delta \\
\Rightarrow \frac{\left|\tilde{S}_{q}\left(p^{\langle 1\rangle}\right)-\tilde{S}_{q}\left(p^{\langle 2\rangle}\right)\right|}{\tilde{S}_{q, \max }}<\varepsilon
\end{array}\right),
\end{aligned}
$$

where $\left\|p^{\langle 1\rangle}-p^{\langle 2\rangle}\right\|_{1}=\sum_{i}\left|p_{i}^{\langle 1\rangle}-p_{i}^{\langle 2\rangle}\right|$ is the distance between probability distributions $\left\{p_{i}^{\langle 1\rangle}\right\}$ and $\left\{p_{i}^{\langle 2\rangle}\right\}$, and $\tilde{S}_{q, \max }$ is the maximum value of eq. (12) realized at equiprobability; i.e. $p_{i}=1 / W$. It has been proved that Tsallis entropy is Lesche stable [20]. By contrast, it is found from eq. (19) that

$$
\begin{aligned}
\frac{\left|\tilde{S}_{q}\left(p^{\langle 1\rangle}\right)-\tilde{S}_{q}\left(p^{\langle 1\rangle}\right)\right|}{\tilde{S}_{q, \max }} & =\frac{\left|S_{q}\left(p^{\langle 1\rangle}\right)-S_{q}\left(p^{\langle 1\rangle}\right)\right|}{S_{q, \max }} \\
& =\frac{\left|\sum_{i}\left(p_{i}^{\langle 1\rangle}\right)^{q}-\sum_{i}\left(p_{i}^{\langle 1\rangle}\right)^{q}\right|}{1-W^{1-q}} .
\end{aligned}
$$

Employing the same technique used in [20], we can easily prove that the generalized entropy of eq. (12) is also Lesche stable.

For incomplete probability normalization, the definition of Lesche stability can be written as [21]

$$
\begin{aligned}
& (\forall \varepsilon>0)(\exists \delta>0) \\
& \left(\begin{array}{l}
\left\|\left(p^{\prime}\right)^{\langle 1\rangle}-\left(p^{\prime}\right)^{\langle 2\rangle}\right\|_{1} \leqslant \delta \\
\Rightarrow \frac{\left|\tilde{S}_{I}\left(p^{\langle 1\rangle}\right)-\tilde{S}_{I}\left(p^{\langle 2\rangle}\right)\right|}{\tilde{S}_{I, \max }}<\varepsilon
\end{array}\right),
\end{aligned}
$$

where $\left\|\left(p^{\prime}\right)^{\langle 1\rangle}-\left(p^{\prime}\right)^{\langle 2\rangle}\right\|_{1}=\sum_{i}\left|\left[\left(p_{i}^{\prime}\right)^{\langle 1\rangle}\right]^{q^{\prime}}-\left[\left(p_{i}^{\prime}\right)^{\langle 2\rangle}\right]^{q^{\prime}}\right|$ is the distance between incomplete probability distributions $\left\{\left(p_{i}^{\prime}\right)^{\langle 1\rangle}\right\}$ and $\left\{\left(p_{i}^{\prime}\right)^{\langle 2\rangle}\right\}$, and $\tilde{S}_{I, \max }$ is the maximum value of eq. (18). From

$$
\begin{aligned}
\frac{\left|\tilde{S}_{I}\left(\left(p^{\prime}\right)^{\langle 1\rangle}\right)-\tilde{S}_{I}\left(\left(p^{\prime}\right)^{\langle 2\rangle}\right)\right|}{\tilde{S}_{I, \max }} & =\frac{\left|S_{I}\left(\left(p^{\prime}\right)^{\langle 1\rangle}\right)-S_{I}\left(\left(p^{\prime}\right)^{\langle 2\rangle}\right)\right|}{S_{I, \max }} \\
& =\frac{\left|\sum_{i}\left(p_{i}^{\prime}\right)^{\langle 1\rangle}-\sum_{i}\left(p_{i}^{\prime}\right)^{\langle 2\rangle}\right|}{\left(W^{\prime}\right)^{1-\frac{1}{q^{\prime}}}-1},
\end{aligned}
$$

we draw the same conclusion as stated in [21] that generalized entropy under a condition of incomplete probability normalization is Lesche stable.

\section{Conclusions}

We have shown that Tsallis entropy and incomplete entropy have similar characteristics because the two entropic forms have the same mathematical structure except for one nonextensive factor. However, neither can yield exactly the observed ubiquitous $q$-exponential distribution obtained employing the standard Lagrange multiplier method. Based on complete and incomplete probability normalization conditions, two generalized entropic forms are proposed to meet the experimental observations [15-17]. Both are Lesche stable, which means that they vary continuously with the probability distribution functions and are thus physically meaningful. Using the generalized entropies, it is possible to discuss the thermodynamic properties of the experimental systems mentioned above, and we will explore this topic further in future work.

This work was supported by the National Natural Science Foundation of China (11005041), Natural Science Foundation of Fujian Province (2010J05007), Scientific Research Foundation for the Returned Overseas Chinese Scholars and Basic Science Research Foundation of Huaqiao University (JB-SJ1005).

1 Chen X, Xuan Y M, Han Y G. Investigation of the entropy generation and efficiency of a solar thermophotovoltaic system. Chinese Sci Bull, 2010, 55: 3718-3726

2 Wang L N, Min J C. Thermodynamic analysis of adsorption process at a non-equilibrium steady state. Chinese Sci Bull, 2010, 55: 3619_ 3625

3 Xia S J, Chen L G, Sun F R. Hamilton-Jacobi-Bellman equations and dynamic programming for power-optimization of a multistage heat engine system with generalized convective heat transfer law. Chinese Sci Bull, 2011, 56: 1147-1157

4 Van Den Berg T L, Fanelli D, Leoncini X. Stationary states and fractional dynamics in systems with long-range interactions. Europhys Lett, 2010, 89: 50010

5 Campa A, Chavanis P H, Giansanti A, et al. Dynamical phase transitions in Hamiltonian long-range systems and Tsallis distributions with time dependent index. Phys Rev E, 2008, 78: 040102R

6 Bentes S R, Menezes R, Mendes D A. Long memory and volatility clustering: Is the empirical evidence consistent across stock markets? Physica A, 2008, 387: 3826-3830

7 Du J L. Some dynamical property of the Tsallis distribution from a Fokker-Planck equation. Chin Phys B, 2010, 19: 040501

8 Wang Q A. Incomplete information and fractal phase space. Chaos Solit Fract, 2004, 19: 639-644

9 Tsallis C. Possible generalization of Boltzmann-Gibbs statistics. J Stat Phys, 1988, 52: 479-487

10 Wang Q A. Incomplete statistics: Nonextensive generalizations of statistical mechanics. Chaos Solit Fract, 2001, 12: 1431-1437

11 Huang Z F, Lin B H, Chen J C. A new expression of the probability distribution in incomplete statistics and fundamental thermodynamic relations. Chaos Solit Fract, 2009, 40: 1277-1281

12 Tsallis C, Mendes R S, Plastino A R. The role of constraints within generalized nonextensive statistics. Physica A, 1998, 261: 534-554

13 Abe S. Generalized molecular chaos hypothesis and H-theorem: Problem of constraints and amendment of nonextensive statistical mechanics. Phys Rev E, 2009, 79: 041116 
14 Abe S. Instability of $q$-averages in nonextensive statistical mechanics. Europhys Lett, 2008, 84: 60006

15 Huang X P, Driscoll C F. Relaxation of 2D turbulence to a metaequilibrium near the minimal enstrophy state. Phys Rev Lett, 1994, 72: 2187-2191

16 Liu B, Goree J. Superdiffusion and non-Gaussian statistics in a driven-dissipative 2D dusty plasma. Phys Rev Lett, 2008, 100: 055003

17 Liu B, Goree J, Feng Y. Non-Gaussian statistics and superdiffusion in a drivendissipative dusty plasma. Phys Rev E, 2008, 78: 046403
18 Ou C J, El Kaabouchi A, Wang Q A, et al. The uncertainty measure for $q$-exponential distribution function. arXiv: 1009.0937v1

19 Wang Q A. Probability distribution and entropy as a measure of uncertainty. J Phys A: Math Theor, 2008, 41: 065004

20 Abe S. Stability of Tsallis entropy and instabilities of Rényi and normalized Tsallis entropies: A basis for $q$-exponential distributions. Phys Rev E, 2002, 66: 046134

21 Ou C J, El Kaabouchi A, Chen J C, et al. Stability of incomplete entropy and incomplete expectation. Physica A, 2009, 388: 1813-1817

Open Access This article is distributed under the terms of the Creative Commons Attribution License which permits any use, distribution, and reproduction in any medium, provided the original author(s) and source are credited. 